\title{
BMJ Open Evaluating the effectiveness of home exercise programmes using an online exercise prescription tool in children with cerebral palsy: protocol for a randomised controlled trial
}

\author{
Rowan W Johnson, ${ }^{1}$ Sian A Williams, ${ }^{2}$ Daniel F Gucciardi, ${ }^{2}$ Natasha Bear, ${ }^{3}$ \\ Noula Gibson ${ }^{1,2,3}$
}

To cite: Johnson RW, Williams SA, Gucciardi DF, et al. Evaluating the effectiveness of home exercise programmes using an online exercise prescription tool in children with cerebral palsy: protocol for a randomised controlled trial. BMJ Open 2018;8:e018316. doi:10.1136/ bmjopen-2017-018316

- Prepublication history for this paper is available online. To view these files, please visit the journal online (http://dx.doi. org/10.1136/bmjopen-2017018316).

Received 19 June 2017 Revised 30 November 2017 Accepted 6 December 2017

Check for updates

${ }^{1}$ Therapy and Health Services, Ability Centre, Perth, Western Australia, Australia

${ }^{2}$ School of Physiotherapy and Exercise Science, Curtin University, Perth, Western Australia, Australia ${ }^{3}$ Department of Physiotherapy, Princess Margaret Hospital for Children, Child and Adolescent Health Service, Perth, Western Australia, Australia

Correspondence to Rowan W Johnson; rowan.johnson@abilitycentre. com.au

\section{ABSTRACT}

Introduction Children with cerebral palsy (CP) and other neurodevelopmental disabilities often receive a home programme of exercises to assist in reaching their therapy goals. Adherence to exercise programmes is necessary to attain the level of practice required to achieve goals; however, adherence can be difficult to accomplish. In this paper, we describe the protocol for a randomised controlled trial to evaluate the effectiveness of delivering a home exercise programme to school-age children with disabilities using Physitrack, an online exercise prescription tool with a website or app interface. Methods and analysis Participants aged $6-17$ years, with $\mathrm{CP}$ or other neurodevelopmental disabilities, receiving community physiotherapy services in Western Australia, will be recruited. Participants will be stratified by age and functional mobility and randomised to either the intervention group, who will complete an 8-week home exercise programme using Physitrack, or the control group, who will complete an 8-week exercise programme without Physitrack. Researcher blinding to group allocation, and participant blinding to outcome, will be maintained. The primary outcome measures are adherence to the home exercise programme with weekly collection of home exercise logs; achievement of individualised goals by phone interview before and after intervention; and correctness of exercise performance by collection and analysis of videos of participants performing home exercises. Secondary outcome measures include enjoyment of physical activity, confidence to complete exercise programme, preferred method of delivery of programme and usability of Physitrack. A sample size of 58 participants will be necessary to see an effect on home programme adherence. Data will be analysed using the intention-to-treat principle.

Ethics and dissemination Ethical approval was obtained from Curtin University Human Research Ethics Committee in July 2016 (10391). Outcomes will be disseminated through publication in peer-reviewed journals and presentations at scientific conferences.

Trial registration number ACTRN12616000743460; Preresults.

\section{Strengths and limitations of this study}

- This study is a randomised controlled trial comparing adherence to home exercise programmes among school-age children with disabilities across two delivery methods used by physiotherapists (conventional vs an online exercise prescription tool).

- Achievement of individualised goals will be measured using a tool with established reliability and validity evidence in past work.

- Researcher and statistician blinding to group allocation will be maintained.

- It is impossible to blind participants to intervention.

- Measuring the quality of exercise performance in individualised exercise programmes is challenging; the measure identified for this purpose has not yet been formally evaluated.

\section{INTRODUCTION}

In children with cerebral palsy $(\mathrm{CP})$, the need for intense practice of therapy activities to promote positive adaptations through neuroplasticity has been identified. ${ }^{12}$ Intensity of interventions for children with CP refers to the frequency and duration of the training session and the duration of the training period. ${ }^{3}$ A systematic review by Sakzewski et $a l^{4}$ determined that intensive goal-directed interventions are more effective than standard therapy and that the intensity of therapy appears to be closely related to clinical outcomes. Tinderholt Myrhaug et a concluded that the intensity of therapy may be as important as the type and context of training.

Exercise programmes delivered in the home environment complement direct intervention and help children with $\mathrm{CP}$ to achieve the necessary intensity of practice to effect 
outcome. ${ }^{4}{ }^{6}$ Home exercise programmes can account for $50 \%-80 \%$ of the total therapy received, with direct intervention with the therapist present making up the remainder. ${ }^{4}$ As well as increased intensity of practice, other benefits of home programmes for children with CP include increased involvement of parents in goal setting and training and improved general education about the health condition. ${ }^{7}$

Several challenges in the effective use of home exercise programmes among children with CP have been identified in the literature, in particular adherence to exercise programmes, with researchers investigating parental experience and recommending strategies to address adherence issues. ${ }^{8-11}$ Some of the recommended strategies include but are not limited to: developing individualised programmes, respecting family preferences and routines, providing feedback to build confidence, using a collaborative decision-making process, prescribing a small number of exercises, provision of required equipment and educating parents in providing emotional and physical support. ${ }^{8-11}$ However, there is a paucity of research on the effectiveness of these recommendations.

Another challenge identified in home programmes for children with $\mathrm{CP}$ is that exercise performance is less controllable than in face-to-face sessions, ${ }^{5}$ meaning that a child may attempt prescribed exercises, yet they may not be performed correctly. Schoo et al ${ }^{12}$ measured the Correctness of Exercise Performance (COEP) ${ }^{13}$ in their study investigating the mode of home exercise instruction among older adults with osteoarthritis. They found that, following face-to-face instruction, the addition of audiotapes or videotapes did not provide further benefit to the provision of exercises brochures in terms COEP scores. ${ }^{12}$ The correctness of exercise performance when carrying out a programme at home has not been investigated in children with CP and other neurodevelopmental disabilities, nor has the use of audiovisual cues, warranting investigation of home programme performance in this population.

Adherence to exercise prescription has been reviewed in adults with chronic health conditions, ${ }^{14}$ but there has been little measurement of adherence to home programmes in children with CP reported in the literature. Where there has been measurement of adherence in children with $\mathrm{CP}$, it has been in small numbers of participants ( $n=9$ to $n=10$ ), and there has been insufficient detail in the reporting of adherence measures. ${ }^{15-17}$ Furthermore these studies do not compare the effect of different exercise programme delivery strategies on adherence, therefore they do not inform clinical practice on this issue. ${ }^{15-17}$ A preceding study by Law $e a^{18}$ in children with spastic CP perhaps gives the best indication of challenges regarding adherence to home programmes. This randomised crossover trial of 50 participants, all receiving a home programme, found that exercise log books were only completed on $68 \%$ of total prescribed exercise days. Of the days that records were kept by parents, full programme completion was seen on only
$58 \%$ of recorded exercise days, with partial programme completion on $30 \%$ and no exercise completion on $13 \%$ of recorded exercise days. This study indicates both the challenge therapists and families of children with CP face with adherence to home programmes and the challenge of accurately measuring and reporting this type of data.

One strategy used recently to improve adherence and performance is the use of technology. The benefits of using technology to improve adherence have been tested in adults, demonstrating its potential to motivate adults to participate in exercise, ${ }^{12}{ }^{19} 20$ but has yet to be investigated in children. Factors affecting adherence in adult populations or populations with different health conditions may not be transferable to paediatric populations with neurological conditions where intensity of intervention is important to effect neuroplastic adaptations and affect outcomes. Thus, there remains a need to test the effectiveness of technological approaches for enhancing adherence to exercise programmes among children with CP or other neurodevelopmental disabilities.

\section{Objectives}

The primary objectives of this study are, in children (aged 6-17 years) with CP and other neurodevelopmental disabilities, to evaluate the effectiveness of an online exercise prescription tool for improving:

1. children's adherence to physiotherapist prescribed exercise programmes

2. children's achievement of individualised goals

3. children's performance of prescribed exercises.

The secondary objectives of this study are to evaluate the effectiveness of an online exercise prescription tool for improving:

4. children's enjoyment of exercise and confidence to exercise

5. children's and parents'/caregivers' satisfaction in the delivery of the exercise programme.

\section{METHODS AND ANALYSIS Trial design}

This study is a parallel-design, participant-blinded (to outcome), researcher-blinded, two-arm pragmatic randomised controlled trial (RCT) with an 8-week intervention period. Both groups will receive an individualised home exercise programme. The intervention group will be provided with a programme on the Physitrack website and app. The control group will be provided with a programme using conventional, paper-based methods. The trial has been registered prospectively with the Australian New Zealand Clinical Trials Registry (ACTRN12616000743460), prior to participant recruitment. Important protocol modifications will be communicated to the trial registry.

\section{Participants and eligibility criteria}

Children aged 6-17 years, with CP or other neurodevelopmental disabilities, living in Western Australia, will be recruited. Inclusion and exclusion criteria are shown in 


\section{Box Eligibility criteria}

\section{Inclusion criteria}

- Diagnosis: cerebral palsy or other neurodevelopmental disabilities. Any childhood diagnosis that leads to physical impairment and disability may be included.

- Age: children aged 6-17 years.

- Family have agreed to having a home exercise programme.

- Physiotherapist determines that the child has the cognitive ability to follow an exercise programme in either written or electronic format, with or without support from their parents.

- Families have access to the internet in their home and can access Physitrack

- Participants and their parents/guardians are fluent in the English language.

\section{Exclusion criteria}

- Has orthopaedic surgery scheduled during the 8-week study period.

- Has another significant medical intervention or inpatient stay that will affect ability to carry out a home exercise programme.

- Has casting intervention scheduled during 8-week study period.

- Receiving an intensive intervention service (frequency greater than two times per week) during the 8-week study period.

box. Exclusion criteria are events that may limit a child's ability to follow a home programme. Many children with $\mathrm{CP}$ regularly have botulinum neurotoxin type A (BoNTA); we do not consider this treatment as an exclusion criterion, because it is usual practice to have a home programme prescribed following BoNTA injections.

\section{Sample size}

An online exercise prescription tool has not yet been studied with this population. Using the published data from a web-delivered intervention that measured adherence $^{21}$ to detect a difference in adherence of $51 \%$ in the control and $85 \%$ in the treatment group, with at least $80 \%$ power and significance level of 0.05 , we require 29 children per group (58 total) using a two-tailed test. To allow for $15 \%$ attrition, our aim is to recruit 33 per group (66 in total).

\section{Recruitment}

Physiotherapists working in community therapy services for children with disabilities in Western Australia will be informed about the study through personal invitation and formal invitation via an email to physiotherapy providers. Physiotherapists to be invited to participate are employed at: Ability Centre-the non-government organisation (NGO) leading the study, other community NGOs, government rural health services and private physiotherapy providers in Western Australia. Physiotherapists who agree to participate will receive training from the principal investigator (RWJ) on how to use Physitrack and to outline their role in the project. Therapists are trained in how to go through the steps of making a Physitrack programme using several training tools: including watching a live video demonstration, discussion with the researcher and provision of documentation as a ready reference guide. Therapists will practice using Physitrack prior to the data collection period and can seek support from the research team or from Physitrack staff directly, if required.

Participating physiotherapists will be asked to identify up to eight children with CP or other neurodevelopmental disabilities on their current caseloads who have an exercise programme as part of their therapy plan and who they think meet the inclusion criteria. Once families have been identified, RWJ will coordinate the mail out of information and consent forms. Those participants who, by assent, agree to be contacted will be called by RWJ and given further oral explanation and opportunity to answer any questions to assist them with making an informed choice to participate. Families can then choose to participate by signing the parent consent forms and returning by mail or email. Children aged 6-11 years are provided with a younger child information sheet and tacit agreement is obtained, and children aged 12-17 years are provided with older-age child information sheets and sign child assent forms. A second recruitment strategy will include using media, including social media to inform the public about the study; if a family expresses interest via this recruitment strategy, we will contact their physiotherapist to determine their suitability including meeting the inclusion criteria.

\section{Randomisation and blinding procedure}

Once written consent to participate has been obtained and before randomisation, participants are mailed and asked to complete the Physical Activity Enjoyment Scale (PACES) in children ${ }^{22}$ and 11-point Numeric Rating Scale (NRS) question about confidence to complete an exercise programme. Participants will then be stratified by a measure of their level of functional mobility as indicated by a rating of their mobility method over a distance of $50 \mathrm{~m}$ using the Functional Mobility Scale (FMS) ${ }^{23}$ and by age (less than 12 years or 12 years and older) and will be assigned randomly to one of two groups using a computerised random number generator.

Principal researchers are blinded to group allocation. This blinding will be initiated by a research associate implementing the randomisation process described above. The associate will notify each participant's treating physiotherapist of group allocation by email, enabling the physiotherapist to implement the home programme directly with the participant while maintaining blinding of the researchers. In addition, the single outcome measure that is measured only among the intervention group, the System Usability Scale (SUS) ${ }^{24}$ (described below), will be collected by that same research associate and held separately until blinding is lifted. The principal researchers and the statistician will continue to be blinded to group allocation, with nominal group names being assigned by the research associate and maintained throughout the analysis of the data. It is impossible to blind the physiotherapists implementing the intervention or the participants to group allocation. However, physiotherapists 
and participants will be blinded to the primary aims of the study, in particular the nature of the primary outcome measures. After their participation in the study is complete, participants will be notified by mail of the specific aims of the study and the outcome measures.

\section{Intervention}

\section{Physitrack}

Physitrack provides the same information to the participant as a conventional exercise programme, and this information is provided via a website or app (Apple iOS or Andriod) and includes videos of how to perform each exercise in the home programme. Videos are either selected from an online exercise library that includes spoken audio instructions or customised videos can be made by the physiotherapist within the app. Exercises are set to a weekly calendar, allowing each exercise to be assigned daily or on selected days of the week. As participants use Physitrack, they are prompted to record exercise completion, and therapists can access this data to track each child's adherence. Use of this feature is encouraged; however, it is not a useful measure of adherence for the study as the control group cannot use this function. Other features available in Physitrack that therapists and participants may choose to use include: setting exercise reminder alerts, monitoring comfort/pain and participant-therapist messaging for feedback and guidance.

\section{Physiotherapist training}

Participating physiotherapists, after completing training, can opt-in to be part of the study. The use of Physitrack involves selecting exercises from a video library, then exercise parameters are set from drop-down boxes and any personalised instructions added. Once all the exercises are selected, they are added to a weekly programme, which is then repeated for 8 weeks. The Physitrack interface is simple to use, and developing an exercise programme on Physitrack follows similar steps as developing a conventional exercise programme. Therefore, we anticipate that physiotherapists will have confidence in using Physitrack particularly with the training provided, practice opportunities and support available. We will measure physiotherapists' experience of Physitrack at the completion of the study (see 'System Usability Scale', below).

\section{Baseline assessments}

After randomisation and just prior to the beginning of the 8-week intervention period, the participant's usual physiotherapist will arrange two appointments. The first appointment is to determine between 1-3 specific goals with the participant that will direct the selection of exercises and then to trial exercises for inclusion in a home exercise programme. Goals are to be targeted within the activity domain of the International Classification of Functioning Disability and Health (ICF) ${ }^{25}$ and will be used in the Canadian Occupational Performance Measure (COPM). ${ }^{26}{ }^{27}$ The ICF defines an activity as the execution of a task or action by an individual ${ }^{25}$ (eg, dressing, maintaining a sitting position or walking on different surfaces). Although the goals are to be targeted at the activity domain, the home exercises selected may address any relevant dimension of the $\mathrm{ICF}^{25}$ including targeting the goal activity directly through specific skill practice or indirectly through exercises that address relevant impairments.

At the second appointment, physiotherapists will review the home exercises and deliver the programme based on the participant's group allocation. The control group will receive their home programme using conventional methods (eg, handwritten, typed or photo-programme). The intervention group will receive their individualised home programme of exercises delivered with Physitrack.

The physiotherapist will explain to the participating parents and children in the intervention group how to use the Physitrack app or website. Further support can be accessed for troubleshooting and advice from their physiotherapist in the first instance, who can then liaise with Physitrack staff further if needed.

\section{Eight-week intervention}

Participants in both groups will receive follow-up clinical support from their physiotherapist in completing the programme (eg, home visits to reinforce exercises), according to the therapist's usual practice and the participant's current therapy plan. The principal investigator will distribute weekly reminders (text message and email) to have the participants return weekly home exercise log sheets. Regardless of how many home exercise sessions have been completed along the way, participants will continue to be monitored over the entire 8 weeks of the study period. Only after this time will any participants be identified as having 'dropped out' (defined as a participant ceasing to return outcome measure forms). The time point at which the participant dropped out, in addition to their adherence prior to the time of dropout, will be recorded for later reporting. At the end of the treatment phase, the children in the control group will be offered Physitrack, so that all children will be offered access to the intervention. For a flow diagram of procedures, see figure 1 .

\section{Outcomes measures and procedures}

The following demographic data will be collected at baseline: age, gender, primary and secondary diagnoses and functional mobility using $\mathrm{FMS}^{2}$ and, for children with CP, the expanded and revised Gross Motor Function Classification System. ${ }^{28}$ In addition, data will be collected on follow-up clinical support for each participant to enable investigation of the impact this has on the outcome measures. For further details about outcome measures, see table 1 .

\section{Adherence}

Adherence has been defined as the extent to which a client completes the active element of intervention. ${ }^{1129}$ Adherence to the prescribed home exercise programme is the primary outcome measure and will be monitored in both groups in two ways: 


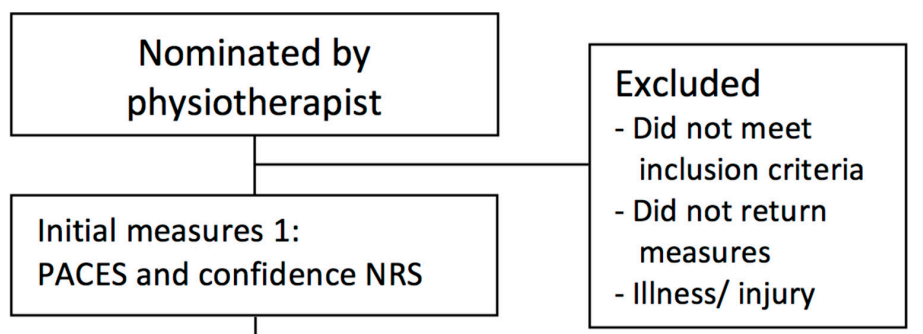

Stratification:

age and mobility level

들
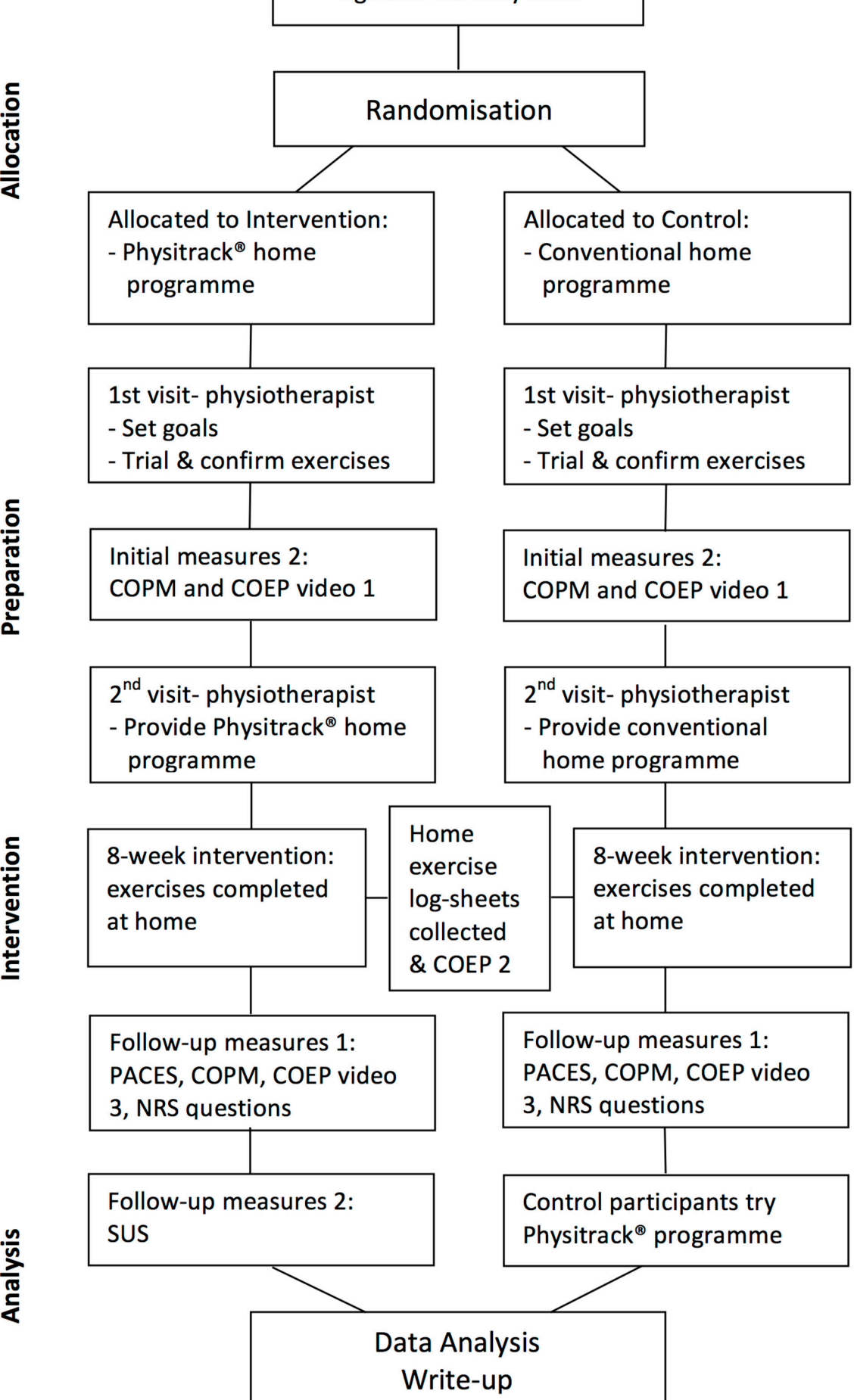

Figure 1 Trial procedures flow diagram. COEP, Correctness of Exercise Performance; COPM, Canadian Occupational Performance Measure; NRS, Numeric Rating Scale; PACES, Physical Activity Enjoyment Scale; SUS, System Usability Scale. 
Table 1 Outcome assessments

\begin{tabular}{|c|c|c|c|}
\hline Outcome & Measure & Validity & Reliability \\
\hline $\begin{array}{l}\text { Self-rated } \\
\text { adherence }\end{array}$ & Numerical Rating Scale (NRS) ${ }^{30}$ & $\begin{array}{l}\text { Adherence measured with 11-point NRS } \\
\text { correlates with adherence measured } \\
\text { from exercise log books }(r=0.62)^{30}\end{array}$ & Not reported \\
\hline Goal achievement & $\begin{array}{l}\text { Canadian Occupational } \\
\text { Performance Measure (COPM), } \\
\text { adapted for children }^{2627}\end{array}$ & $\begin{array}{l}\text { Correlations of change in the adapted } \\
\text { total COPM performance score with Goal } \\
\text { Attainment Scaling (GAS) were all highly } \\
\text { significant }(P<0.05) \text { and in moderate } \\
0.35-0.49 \text { Spearman's correlation } \\
\text { range }^{2627}\end{array}$ & $\begin{array}{l}\text { Alpha coefficient at each } \\
\text { measurement occasion: } \\
\text { Performance:mean } 0.73 \\
\text { Satisfaction: mean } 0.88^{26} 27\end{array}$ \\
\hline $\begin{array}{l}\text { Exercise } \\
\text { performance }\end{array}$ & $\begin{array}{l}\text { Correctness of Exercise } \\
\text { Performance (COEP) })^{1213}\end{array}$ & $\begin{array}{l}\text { 'COEP is a simple tool although } \\
\text { face validity has not been formally } \\
\text { reported'. } 1213\end{array}$ & $\begin{array}{l}\text { Interobserver agreement } \\
\text { reported: Kappa coefficient } \\
0.88 \text {. }\end{array}$ \\
\hline $\begin{array}{l}\text { Enjoyment } \\
\text { ofphysical activity }\end{array}$ & $\begin{array}{l}\text { Physical Activity Enjoyment Scale } \\
\text { in children }{ }^{22}\end{array}$ & $\begin{array}{l}\text { Significantly correlated with task goal } \\
\text { orientation }(r=0.65, P<0.01) \text {, athletic } \\
\text { competence }(r=0.23, P<0.01) \text {, physical } \\
\text { appearance }(r=0.20, P<0.01) \text { and self } \\
\text {-reported physical activity }(r=0.16 \text {, } \\
P<0.01)^{22}\end{array}$ & $\begin{array}{l}\text { Acceptable internal reliability: } \\
\text { alpha coefficient: } 0.87 .^{22}\end{array}$ \\
\hline $\begin{array}{l}\text { Usability of } \\
\text { technology }\end{array}$ & System Usability Scale ${ }^{24}$ & Not reported & Alpha coefficient: $0.85^{24}$ \\
\hline
\end{tabular}

1. Participants will complete an exercise log book that will consist of a single sheet for each week, set out in the table format. In this format, the child or parent can easily record the repetitions, sets and/or exercise time completed against each exercise. Families will be reminded weekly, by text message and email, to return a copy of the completed exercise log sheets. Data from $\log$ books will be used to measure adherence as the number of exercise days, the number of exercises started and number of repetitions completed as a proportion of those prescribed.

2. Self-rated adherence to exercise method, frequency of sessions, number of exercises and exercise duration will be assessed at the end of the 8-week intervention using four statements scored using an 11-point NRS $(0=$ strongly disagree, $10=$ strongly agree $) .^{30}$

\section{Canadian Occupational Performance Measure}

Attainment of up to three goals, set before commencement of the intervention, will be measured using the COPM, modified for use with children. ${ }^{24}$ The $\mathrm{COPM}^{2627}$ is an individualised, criterion referenced measure of performance of a self-selected range of activities. For this study, up to three individualised goals are determined by the family during an informal interview with their usual treating physiotherapist. Then, before and after the intervention period, parents-in consultation with children as they deem appropriate-will self-rate their current level of performance of each goal and satisfaction with their performance during a telephone conversation with RWJ.

\section{Correctness of Exercise Performance}

The COEP ${ }^{13}$ scale grades of the quality of exercise performance according to three criteria:
1. 'The performance has been carried out so well that the goal of the exercise is reached'.

2. 'The exercise is not performed correctly and the goal is not reached, although no negative effect is to be expected'.

3. 'The exercise is carried out incorrectly, the goal is not reached and there is reason to assume that the exercise causes harm'.

Videos of the participants performing prescribed exercises at home will be collected at the beginning of the 8-week intervention period, at the half-way (4week) point and immediately following the end of the intervention period. The videos will be collected at baseline by the participants' usual treating physiotherapist and then by volunteers at the midpoint and endpoint. The videos will be scored on the COEP scale retrospectively through a consensus agreement by two investigators who are blinded to the assessment time point of the video and the group allocation.

\section{Physical Activity and Enjoyment Scale}

The PACES ${ }^{22}$ is a measure of enjoyment of physical activity in children. This questionnaire consists of 16 statements that are scored on a five-point rating scale (1=disagreea lot, $5=$ agree a lot). This will be provided as a paper survey to the participants in both groups, before and after the intervention period.

\section{Numeric Rating Scale}

Before the intervention, one survey question will be asked using an 11-point NRS ( $0=$ strongly disagree, $10=$ strongly agree) about the participant's confidence to complete an exercise programme. ${ }^{30}$ After the completion of the intervention, seven survey questions will be asked using an 11 -point NRS ( $0=$ strongly disagree, $10=$ strongly agree), 
covering confidence to complete the programme, satisfaction with the delivery of the exercise programme and process measures. ${ }^{30}$ Participants will be asked to rank different methods of exercise programme delivery (paper sheet: handwritten; paper sheet: typed sheet; paper sheet: preprinted leaflet; electronic handout emailed to them; and online interactive application on smartphone, tablet or computer), by preference, from 1 to 5 , where 1 was the most preferred option. ${ }^{30}$

\section{System Usability Scale}

The $\mathrm{SUS}^{24}$ was developed as a quick survey to assess the usability of a given product or service. Participating physiotherapists and parents in the treatment group will be questioned about the usability of the Physitrack system using a modified SUS, along with open feedback questions provided to them at the end of the study regarding how they accessed Physitrack and their experience of using it at home.

\section{Data management}

All research data will be stored and managed in accordance with the Australian Code for the Responsible Conduct of Research. Data will be collected on a standardised study data entry form and entered onto the data management system by RWJ. Data entry edit checks (for unusual entries, outliers and duplicates) will be initiated quarterly. Paper copies of assessments will be kept in a locked filing cabinet, for a period of 7 years, after which all paper copies will be destroyed. Survey and behavioural data collected will be deidentified and stored in an electronic format on a secure Curtin University server that is backed-up automatically. All data will be retained for at least 25 years, because it involves an RCT (Western Australian University Sector Disposal Authority). Direct access to the identified data will be restricted to listed authors and contributors; access to deidentified data for external parties will be provided on request (eg, for meta-analysis purposes). Structures such as a data monitoring committee and interim data analysis are not required, because this study does not have serious outcomes in terms of morbidity or mortality, neither does increase risk of any harm to participants.

\section{Data analysis}

Data will be analysed using the intention-to-treat principle. Participant characteristics will be described using means and SD for continuous data, median and IRQs for skewed or ordinal data and frequencies and percentages for categorical data. Adherence will be measured by three variables (days, number of exercises and number of repetitions). Adherence will be calculated as a proportion. For example, the total number of exercises completed divided by the total number of exercises prescribed across the 8-week intervention period. The two groups will be compared using a t-test or the non-parametric equivalence (Mann-Whitney U test) if required. In addition, as adherence can be considered as a count variable, Poisson regression will be used with group allocation entered as an independent variable in the regression equation. This analysis will calculate incident rate ratios and their 95\% CI allowing comparison of the rates between the two groups. The influence of the number of follow-up clinical appointments on adherence outcomes will be examined initially through scatter plots for each group followed by univariate regression analysis. Multivariate regression analysis will then be used to examine the independent contributions of group allocation and number of intervention sessions. The COPM performance and satisfaction scores will be calculated for each participant. The groups will be compared using analysis of covariance, with the baseline variable and group entered into the regression equation. This analysis will calculate the mean change from baseline (and their 95\% CI). A two-point change in score on the COPM is considered to be clinically meaningful. ${ }^{26}$ The proportion of participants in each group that show a two-point change in COPM score will be compared between the two groups using $\chi^{2}$ or Fisher's exact test. Correctness of exercise performance is measured on an ordinal scale, which will be analysed for between group differences using a Mann-Whitney $\mathrm{U}$ test. Statistical significance will be set as $\mathrm{P}<0.05$. All statistical analyses will be performed using STATA V.14.1. Further exploratory analysis will be implemented to supplement primary findings, including comparing the impact of adherence on exercise performance and on self-reported activity performance and satisfaction.

\section{Preparing manuscript}

This document was written following Consolidated Standards of Reporting Trials ${ }^{31}$ guidelines for randomised trials and the Standard Protocol Items: Recommendations for Interventional Trials guidelines ${ }^{32}$ for clinical trial protocols.

\section{Ethics and dissemination}

There are no inherent risks to children participating in this study. The exercise programme will have been identified, discussed and agreed to by the participants' parents with their therapist, as part of the usual delivery of services. The only difference to usual practice for the treatment group is the method of delivery of the exercise programme, that is, using a website or app to access the programme rather than a written programme. The only risk or harm would be from practising the exercises incorrectly so that they may not be achieving the desirable outcome. This potential risk is no greater than that would occur from usual care, and in the Physitrack group this may be minimised further due to the monitoring provided by the Physitrack app.

The principal investigator (RWJ) has worked as a senior clinician, and some of the participants would be known to him. During the period of the study, RWJ will not be involved in direct care of the study participants, and during recruitment, it will be made clear that participation is voluntary and will not affect their care now or 
in the future. Additionally, the participant information sheets for both parents and children, along with parent consent forms and child assent forms, are written clearly so as to support informed consent and autonomy. The principal (RWJ) and supervisory investigator (NG) are employed at the same community NGO as the majority of the participating physiotherapists. Physiotherapist participation in the study is voluntary and an opt-in process; an honest, collaborative partnership is developed between the investigators and physiotherapists throughout the study with the health and well-being of participants and non-participating children as the primary goal.

Procedures for protocol amendments, reporting of adverse events and maintaining confidentiality of information will be followed as per Curtin University Human Research Ethics Committee. Results of this study are to be published in respected, peer-reviewed journals and findings presented at scientific conferences in the field of CP and disability.

\section{DISCUSSION}

Home exercise programmes are a frequently used intervention strategy for physiotherapists working with school-age children with $\mathrm{CP}$ and other neurodevelopmental disabilities. Emerging technology has the potential to assist individuals to complete their home exercise so that they can achieve their therapy goals and maximise participation in community physical activity. Physitrack is an exercise prescription tool that operates across multiple platforms, with innovative features that are unavailable in written or in traditional software-based exercise programmes.

This paper outlines the research protocol of an RCT to evaluate the effectiveness of delivering home exercise programmes for children with disabilities using an online exercise prescription tool in comparison with conventional methods. If effective, Physitrack may provide a novel, cost-effective and enjoyable way of increasing exercise practice and improving performance of home exercise. More broadly, Physitrack may also provide a platform for addressing inequities of intervention delivery due to geographical isolation of people with disabilities in rural and remote areas. If found to be ineffective, this study will still provide valuable data about adherence to, and performance of, individualised home programmes that has not been previously investigated in this population and will inform clinical practice.

\section{Acknowledgements We would like to acknowledge the support of} Non-Government Centre Support (NGCS), Department of Education, Government of Western Australia, which provided a research grant for this study. The authors acknowledge the contribution of an Australian Government Research Training Program Scholarship in supporting this research. We would like to thank Dr Amanda Marie Blackmore (Ability Centre) and Melissa Barone (Ability Centre).

Contributors RWJ conceived the idea for the trial, reviewed literature, identified outcome measures, sought ethical approval and drafted the paper. NG developed the trial concept further, reviewed literature, assisted with selection of outcome measures, ethical approval, developing the paper and provided supervision. SAW and DFG provided supervision around the concept of the trial, literature review, ethics application, the outcome measure collection process and developing the paper. NB provided consultation in statistical methods, performed the power calculation and planned the statistical analysis. AMB participated in the trial concept development, assisted in the grant application, participant randomisation and research process consultation. MB assisted in communications, monitoring the trial process and data entry.

Funding This work is supported by a research grant from the study Non-Government Centre Support (NGCS), Department of Education, Government of Western Australia, and in-kind support from Ability Centre, Western Australia. NGCS does not hold influence over study design; collection, management, analysis and interpretation of data; writing of the report; and the decision to submit the report for publication.

Competing interests None declared.

Patient consent Not required.

Ethics approval Ethical approval was attained from Human Research Ethics Committee, Curtin University, Perth, Western Australia (ref. number 10391).

Provenance and peer review Not commissioned; externally peer reviewed.

Open Access This is an Open Access article distributed in accordance with the Creative Commons Attribution Non Commercial (CC BY-NC 4.0) license, which permits others to distribute, remix, adapt, build upon this work non-commercially, and license their derivative works on different terms, provided the original work is properly cited and the use is non-commercial. See: http://creativecommons.org/ licenses/by-nc/4.0/

(c) Article author(s) (or their employer(s) unless otherwise stated in the text of the article) 2018. All rights reserved. No commercial use is permitted unless otherwise expressly granted.

\section{REFERENCES}

1. Kleim JA, Jones TA. Principles of experience-dependent neural plasticity: implications for rehabilitation after brain damage. J Speech Lang Hear Res 2008;51:S225-39.

2. Novak I, Berry J. Home program intervention effectiveness evidence. Phys Occup Ther Pediatr 2014;34:384-9.

3. Palisano RJ, Murr S. Intensity of therapy services: what are the considerations? Phys Occup Ther Pediatr 2009;29:107-12.

4. Sakzewski L, Ziviani J, Boyd RN. Efficacy of upper limb therapies for unilateral cerebral palsy: a meta-analysis. Pediatrics 2014;133:e175.

5. Tinderholt Myrhaug H, Østensjø S, Larun L, et al. Intensive training of motor function and functional skills among young children with cerebral palsy: a systematic review and meta-analysis. BMC Pediatr 2014;14:1.

6. Novak I, Cusick A. Home programmes in paediatric occupational therapy for children with cerebral palsy: where to start? Aust Occup Ther J 2006;53:251-64.

7. Novak I, Cusick A, Lannin N. Occupational therapy home programs for cerebral palsy: double-blind, randomized, controlled trial. Pediatrics 2009;124:e606.

8. Lillo-Navarro C, Medina-Mirapeix F, Escolar-Reina P, et al. Parents of children with physical disabilities perceive that characteristics of home exercise programs and physiotherapists' teaching styles influence adherence: a qualitative study. J Physiother 2015;61:81-6.

9. Novak I. Parent experience of implementing effective home programs. Phys Occup Ther Pediatr 2011;31:198-213.

10. Peplow UC, Carpenter C. Perceptions of parents of children with cerebral palsy about the relevance of and adherence to, exercise programs: a qualitative study. Phys Occup Ther Pediatr 2013;33:285-99.

11. Taylor NF, Dodd KJ, McBurney H, et al. Factors influencing adherence to a home-based strength-training programme for young people with cerebral palsy. Physiotherapy 2004;90:57-63.

12. Schoo AMM, Morris ME, Bui QM. The effects of mode of exercise instruction on compliance with a home exercise program in older adults with osteoarthritis. Physiotherapy 2005;91:79-86.

13. Friedrich $M$, Cermak $T$, Maderbacher $P$. The effect of brochure use versus therapist teaching on patients performing therapeutic exercise and on changes in impairment status. Phys Ther 1996;76:1082-8.

14. Jordan JL, Holden MA, Mason EE, et al. Interventions to improve adherence to exercise for chronic musculoskeletal pain in adults. Cochrane Database Syst Rev 2010:CD005956.

15. Dodd KJ, Taylor NF, Graham HK. A randomized clinical trial of strength training in young people with cerebral palsy. Dev Med Child Neurol 2003;45:652-7. 
16. Fragala-Pinkham MA, Haley SM, Rabin J, et al. A fitness program for children with disabilities. Phys Ther 2005;85:1182-200.

17. Katz-Leurer $\mathrm{M}$, Rotem $\mathrm{H}$, Keren $\mathrm{O}$, et al. The effects of a 'homebased' task-oriented exercise programme on motor and balance performance in children with spastic cerebral palsy and severe traumatic brain injury. Clin Rehabil 2009;23:714-24.

18. Law M, Russell $\mathrm{D}$, Pollock $\mathrm{N}$, et al. A comparison of intensive neurodevelopmental therapy plus casting and a regular occupationa therapy program for children with cerebral palsy. Dev Med Child Neurol 1997;39:664-70.

19. Silveira P, van de Langenberg R, van Het Reve E, et al. Tabletbased strength-balance training to motivate and improve adherence to exercise in independently living older people: a phase II preclinical exploratory trial. J Med Internet Res 2013;15:e159.

20. Zulman DM, Damschroder LJ, Smith RG, et al. Implementation and evaluation of an incentivized Internet-mediated walking program for obese adults. Trans/ Behav Med 2013;3:357-69.

21. Breitenstein SM, Fogg L, Ocampo EV, et al. Parent use and efficacy of a self-administered, tablet-based parent training intervention: a randomized controlled trial. JMIR Mhealth Uhealth 2016;4:e36.

22. Moore JB, Yin Z, Hanes J, et al. Measuring enjoyment of physical activity in children: validation of the physical activity enjoyment scale. $J$ Appl Sport Psychol 2009;21(Suppl 1):S116-29.

23. Graham HK, Harvey A, Rodda J, et al. The Functional Mobility Scale (FMS). J Pediatr Orthop 2004;24:514-20.
24. Bangor A, Kortum PT, Miller JT. An empirical evaluation of the system usability scale. Int J Hum Comput Interact 2008;24:574-94.

25. World Health Organisation. International classification of functioning, disability and health: children \&amp; youth version. Geneva: World Health Organization, 2007.

26. Law M, Baptiste S, Carswell A, et al. Canadian Occupational Performance Measure (COPM): Canadian Association of Occupational Therapists, 2014.

27. Cusick A, Lannin NA, Lowe K. Adapting the canadian occupational performance measure for use in a paediatric clinical trial. Disabil Rehabil 2007;29:761-6.

28. Palisano RJ, Rosenbaum P, Bartlett $\mathrm{D}$, et al. Content validity of the expanded and revised gross motor function classification system. Dev Med Child Neurol 2008;50:744-50.

29. Chen CY, Neufeld PS, Feely CA, et al. Factors influencing compliance with home exercise programs among patients with upper-extremity impairment. Am J Occup Ther 1999;53:171-80.

30. Bennell K, Charlotte M, Lonsdale C, et al. Patient Adherence to Home Exercise Programs (PANTHER): randomised controlled trial protocol the university of melbourne, 2015.

31. Schulz KF, Altman DG, Moher D. CONSORT 2010 statement: updated guidelines for reporting parallel group randomized trials. Ann Intern Med 2010;152:726.

32. Chan AW, Tetzlaff JM, Altman DG, et al. SPIRIT 2013 statement: defining standard protocol items for clinical trials. Ann Intern Med 2013;158:200. 